\title{
CASI CONFERMATI DI CANCRO CERVICALE IN BRASILE TRA IL 2010 E IL 2014
}

\section{ARTICOLO ORIGINALE}

FARIAS, Rafaela Santos ${ }^{1}$, FACCO, Lucas², FECURY, Amanda Alves³, ARAÚJO, Maria Helena Mendonça de ${ }^{4}$, OLIVEIRA, Euzébio de ${ }^{5}$, DENDASCK, Carla Viana ${ }^{6}$, SOUZA, Keulle Oliveira da ${ }^{7}$, DIAS, Claudio Alberto Gellis de Mattos ${ }^{8}$

\section{FARIAS, Rafaela Santos. Et. Casi confermati di cancro cervicale in Brasile tra il}

2010 e il 2014. Revista Científica Multidisciplinar Núcleo do Conhecimento. Anno 05, Ed. 11, Vol. 25, pp. 93-104. novembre 2020. ISSN: 2448-0959, Link di accesso: https://www.nucleodoconhecimento.com.br/salute/cancro-cervicale, DOI: 10.32749/nucleodoconhecimento.com.br/salute/cancro-cervicale

\section{RIEPILOGO}

II Pap smear (PCCU) è un esame ginecologico eseguito nelle donne al fine di rilevare danni alla cervice. I cambiamenti possono essere osservati come un disturbo tissutale che lo costituisce. L'obiettivo di questo lavoro è mostrare casi confermati di cancro cervicale in Brasile tra il 2010 e il 2014. La ricerca è stata condotta con i dati presi da DATASUS (http://datasus.saude.gov.br). Quando ci sono buone prestazioni per prevenire una malattia considerata grave, il risultato è soddisfacente ed efficace. Attraverso i risultati ottenuti analizzando i dati tra gli anni 2010 e 2014,

\footnotetext{
${ }^{1}$ Tecnica mineraria, come risultato dell'Istituto Federale di Amapá (IFAP).

${ }^{2}$ Studente del Corso di Medicina dell'Università Federale di Amapá (UNIFAP).

${ }^{3}$ Biomedicale, Dottorato di Ricerca in Malattie Tropicali, Professore e ricercatore del Corso di Medicina dell'Università Federale di Amapá (UNIFAP).

${ }^{4}$ Medico, professore e ricercatore del corso di medicina dell'Università federale di Amapá (UNIFAP).

${ }^{5}$ Biologo, Dottore di Ricerca in Malattie Topiche, Professore e ricercatore del Corso di Educazione Fisica dell'Università Federale di Pará (UFPA).

${ }^{6}$ Teologo, Dottore di Ricerca in Psicoanalisi, ricercatore presso il Centro di Ricerca e Studi Avanzati - CEPA.

${ }^{7}$ Sociologo, studente magistrale in Studi Antropici in Amazzonia, Membro del Gruppo di Ricerca "Laboratorio di Educazione, Ambiente e Salute" (LEMAS/UFPA).

${ }^{8}$ Biologo, Dottore di Ricerca in Teoria e Comportamento, Professore e ricercatore del Graduate Program in Professional and Technological Education (PROFEPT), Istituto Federale di Amapá (IFAP).
}

RC: 67820

Disponível em: https://www.nucleodoconhecimento.com.br/salute/cancro-cervicale 
rispettivamente, si conclude che nel corso degli anni la ricerca del test è gradualmente aumentata, ma alcuni aspetti sono persistito, come la vergogna di eseguire il test pcc, la paura, il dolore, tra gli altri. Tuttavia, i risultati hanno mostrato un alto tasso di test nella regione sudorientale e un tasso più basso nel nord, con scarsa diffusione e scarsa consapevolezza per le donne nella regione settentrionale. Pertanto, la diffusione, la consapevolezza e le prestazioni dell'esame citopatologico sono molto importanti per la prevenzione del cancro cervicale, che oggi è la principale causa di mortalità tra le donne brasiliane

Parole chiave: Epidemiologia, cancro cervicale, PCCU, Citopatologia.

\section{INTRODUZIONE}

Vengono eseguiti test preventivi per identificare possibili malattie e curarle nei loro inizi (BRASIL, 2013). II Pap smear (PCCU) è un esame ginecologico eseguito nelle donne al fine di rilevare danni alla cervice, che possono diventare dannosi per la salute (TEIXEIRA et al., 2015). Questo test viene utilizzato per scoprire i primi cambiamenti nella cervice. Aiuta nella diagnosi precoce del cancro femminile e può essere trattato nel suo stato iniziale, evitando così un aumento del tasso di mortalità tra le donne (ARAÚJO et al., 2016). Questo esame deve essere eseguito da donne che hanno una vita sessualmente attiva o che hanno più di 18 anni. Si basa sulla raccolta e l'analisi di campioni cervicali per identificare il cancro (BARBER et al., 2009).

I cambiamenti possono essere osservati come un disturbo tissutale che costituisce la cervice. Questo disturbo può essere suddiviso in gradi. II grado 1 (I) si verifica quando si verifica un leggero disturbo delle cellule, compromettendo piccole parti del tessuto. Quando questo disturbo cellulare raggiunge fino a tre quarti del tessuto, è considerato nel grado 2 (II). Nel grado 3 (III) si osserva che la disposizione delle cellule è totalmente disordinata, identificando il cosiddetto cancro cervicale (MENETRIER et al., 2016).

$\mathrm{RC}: 67820$

Disponível em: https://www.nucleodoconhecimento.com.br/salute/cancro-cervicale 
II numero di esami PCCU eseguiti in Brasile nel 2010 è stato di circa 10 milioni di esami all'anno. Questa quantità di pap smear scan variava tra le regioni brasiliane. Nella regione settentrionale sono stati eseguiti 603.738 (seicentotremila settecentotrentaotto) esami. La regione nord-orientale ha effettuato 2.616.344 (due milioni e seicentodiciduemilatrecentoquarantaquattro) esami. Nel Midwest furono eseguiti 745.875 (Settecentoquarantacinquemilaottocentosettantacinque). Nel sudest sono state esaminate 4.703 .400 (quattro milioni settecentotremilaquattrocento) persone. Nella regione meridionale, 1.606.119 (un milione e seicentoseimilaciannove) esami (BORTOLON et al., 2012).

II numero di esami PCCU eseguiti nel 2010 nella regione settentrionale del Brasile varia a seconda dello stato che lo compone. Ad Amapá furono eseguiti 19.657 (diciannovemilaseicentocinquanta) esami. Ad Acri furono eseguiti 53.754 (Cinquantatremila settecentocinquantaquattro). In amazonas 134.268 (Centotrentaquattromila duecentosessantaotto) esami eseguiti. Nello stato di Pará, 191.298 (Centonovemilanovemilavantotto) esami. In Rondônia sono stati eseguiti 92.085 (novantanovemila e ottantasi due) esami. Nello stato di Roraima sono stati eseguiti 28.614 (Ventottomilaseicentoquattordici) esami. A Tocantins sono stati eseguiti 84.062 (ottantaquattromila e sessantadue) test (BORTOLON et al., 2012).

\section{GOL}

Mostra casi confermati di cancro cervicale in Brasile tra il 2010 e il 2014.

\section{METODO}

La ricerca è stata condotta con i dati presi da DATASUS

(http://datasus.saude.gov.br) seguendo i seguenti passaggi: in primo luogo, è stata selezionata la scheda "accesso alle informazioni", seguita dall'opzione "informazioni sulla salute (TABNET)" subito dopo la sotto-opzione "Epidemiologica e Morbilità" dopo aver effettuato l'accesso al gruppo di opzioni "Cancro cervicale e mammario (SISCOLO/SISMAMA). Successivamente, è stata selezionata l'icona "Cancro

$\mathrm{RC}: 67820$

Disponível em: https://www.nucleodoconhecimento.com.br/salute/cancro-cervicale 
cervicale e mammario - dal 2000". È stata aperta la scheda "Siscolo 4.00 o superiore" ed è stata selezionata l'opzione "Esame citopatologico Cervico-vaginale e microflora-procedura 12.011.01-0". Quindi cliccato su "Brasile / regioni". Per la raccolta dei dati, è stata selezionata l'opzione "Anno di competenza" nel campo della linea. Nel campo del contenuto è stata selezionata l'opzione "Numero di esami". Nel campo colonna le opzioni erano "Non attivo"; "anno di competenza"; Anno di competenza"; "Reg.residência"; "Scolarizzazione"; "Colore/Razza"; "Entro limiti normali"; "Interv raccoglie"; "Risultato Interv"; "Citopatolo.Precedente"; e "Últ Time.Prevenire. Per tutte le opzioni, i dati sono stati raccolti dal 2010 al 2014. L'opzione "Mese/Anno di competenza" è stata selezionata anche nel campo colonna nel campo colonna e nel campo del contenuto l'opzione "Numero di esami" per gli stessi anni. I dati sono stati compilati all'interno dell'applicazione Excel, un componente della suite Microsoft Corporation Office. La ricerca bibliografica è stata condotta in articoli scientifici, utilizzando per la ricerca computer del laboratorio informatico dell'Istituto Federale di Educazione, Scienza e Tecnologia di Amapá, Câmpus Macapá, situato a: Rodovia BR 210 KM 3, s/n - Bairro Brasil Novo, CEP: 68.909-398, Macapá, Amapá, Brasile.

\section{RISULTATI}

La figura 1 mostra la percentuale di scansioni pap smear (PCCU) eseguite in Brasile tra il 2010 e il 2014. Tra il 2010 e il 2013 il numero di test è rimasto praticamente stabile. Nel 2014, i dati mostrano che c'è stata una forte diminuzione dello svolgimento degli esami nel paese.

RC: 67820

Disponível em: https://www.nucleodoconhecimento.com.br/salute/cancro-cervicale 
Figura 1 Mostra la percentuale di scansioni pap smear (PCCU) eseguite in Brasile tra il 2010 e il 2014.

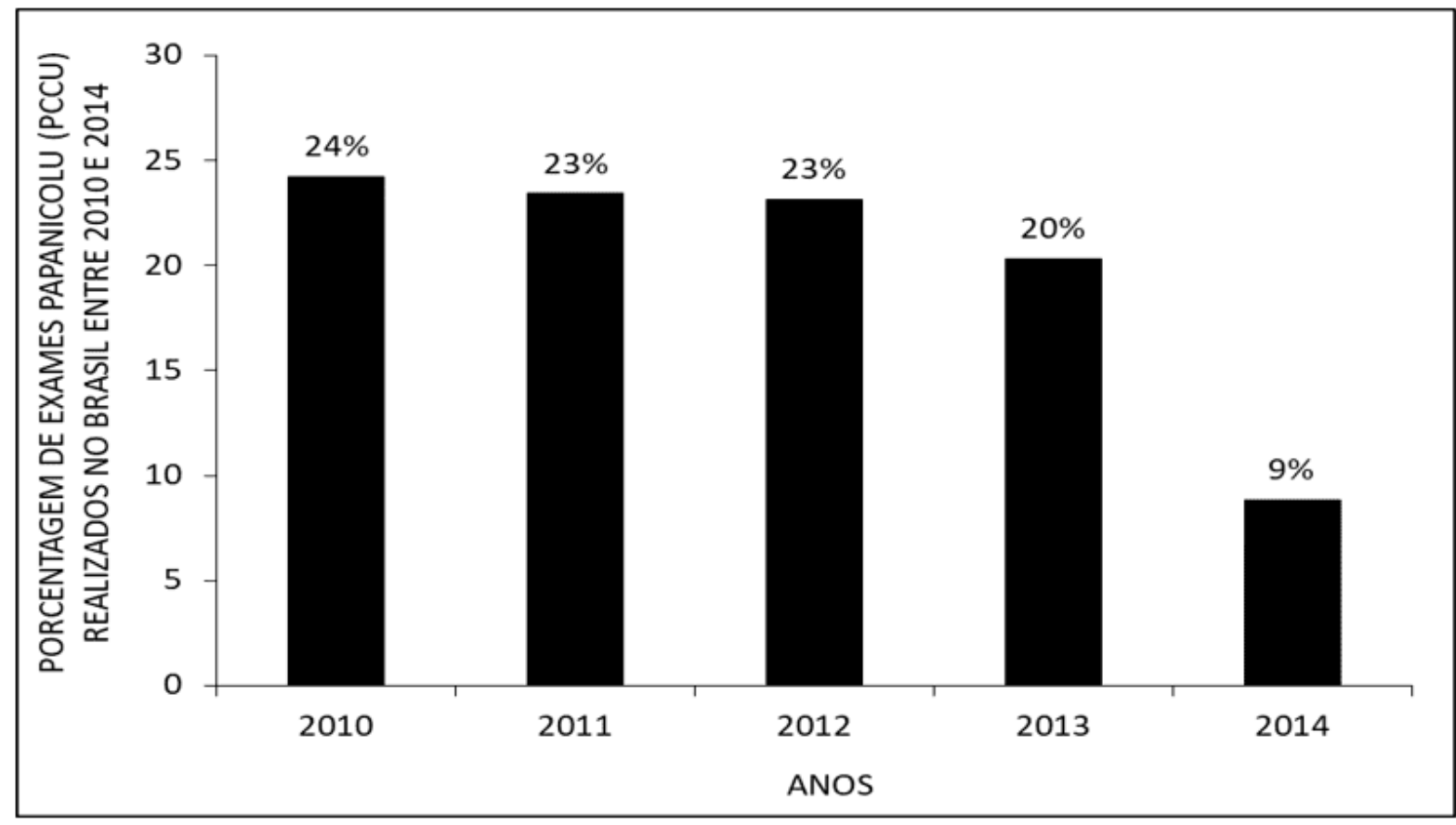

La figura 2 mostra la percentuale di scansioni pap smear eseguite in Brasile tra il 2010 e il 2014, per regioni del paese. Mostrando un tasso più elevato di test eseguiti nella regione sud-orientale e un numero inferiore di test rispettivamente nelle regioni nord-est, sud, midwest e nord.

RC: 67820

Disponível em: https://www.nucleodoconhecimento.com.br/salute/cancro-cervicale 
Figura 2 Mostra la percentuale di scansioni pap smear (PCCU) eseguite in Brasile tra il 2010 e il 2014, per regioni del paese.

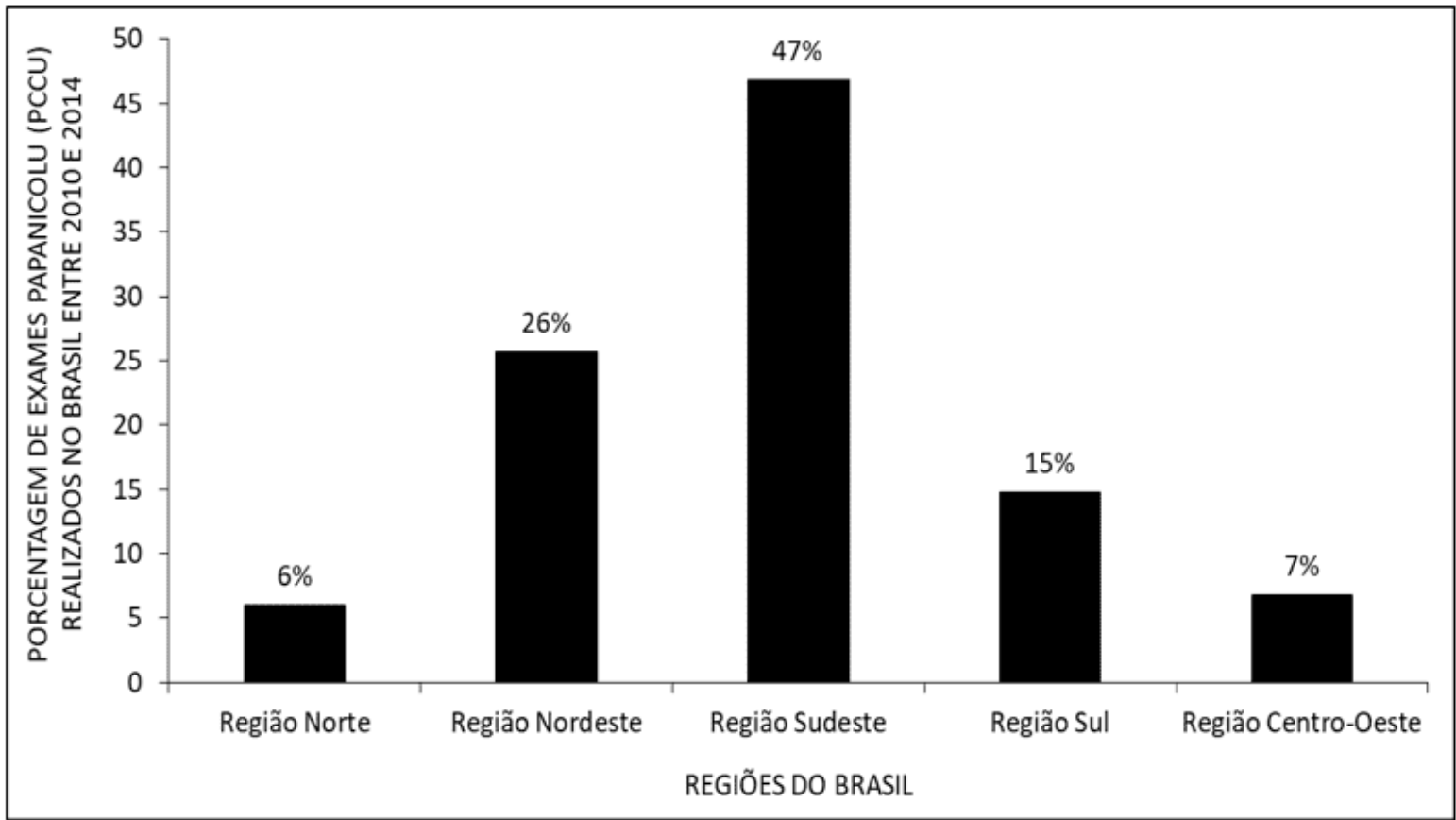

La figura 3 mostra la percentuale di scansioni pap smear (PCCU) eseguite in Brasile tra il 2010 e il 2014, in base alla fascia d'età. I dati mostrano che il maggior numero di test viene eseguito tra le donne di età compresa tra 30 e 39 anni. II più piccolo è nella fascia d'età delle donne fino a 19 anni.

RC: 67820

Disponível em: https://www.nucleodoconhecimento.com.br/salute/cancro-cervicale 
Figura 3 Mostra la percentuale di scansioni pap smear (PCCU) eseguite in Brasile tra il 2010 e il 2014. in base alla fascia d'età.

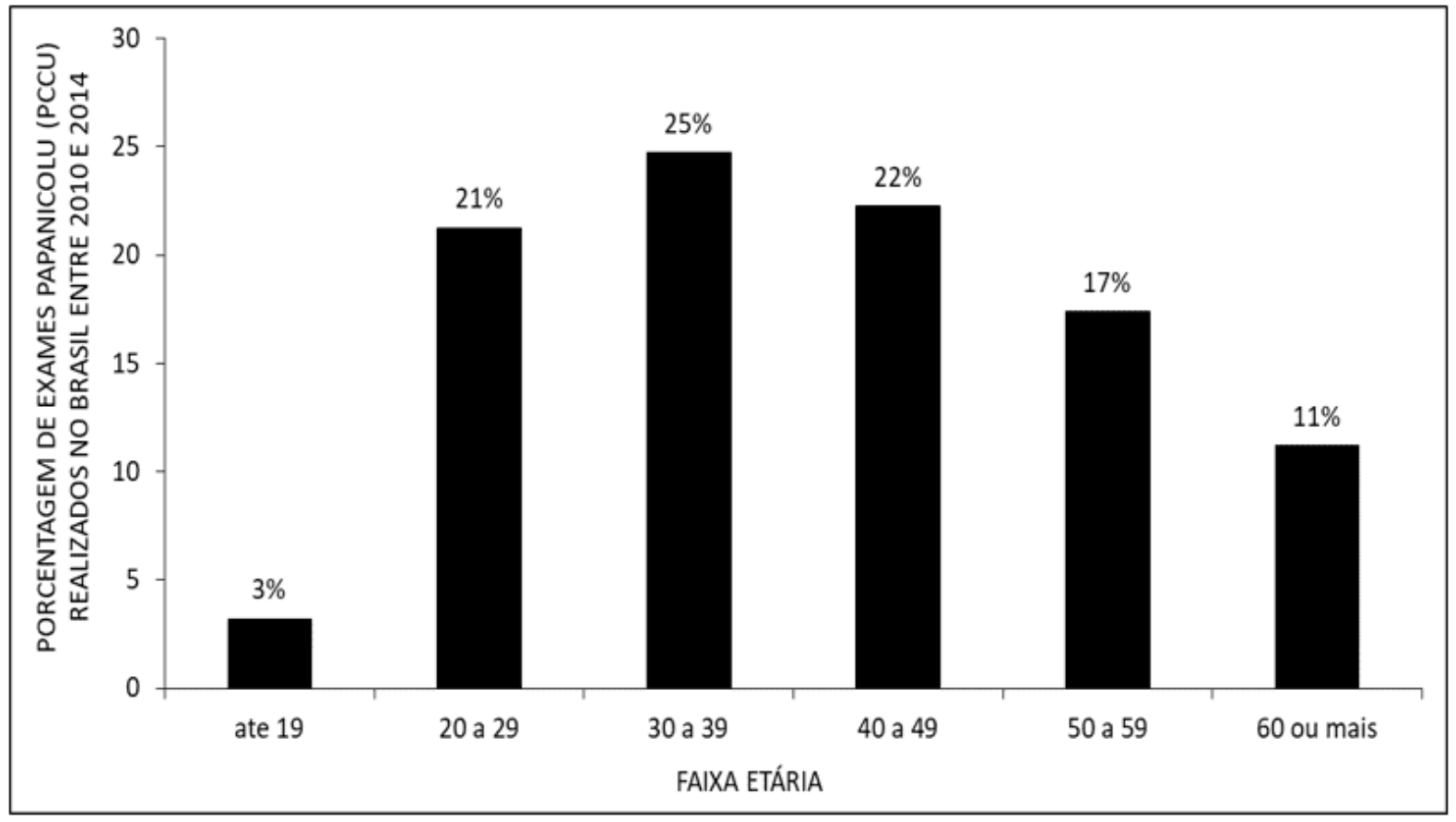

La figura 4 mostra la percentuale di scansioni pap smear (PCCU) eseguite in Brasile tra il 2010 e il 2014, mediante scolarizzazione. Mostrando che la maggior parte delle donne che fanno l'esame hanno una scuola elementare incompleta. Mentre l'importo più ridotto ha completato l'istruzione superiore.

RC: 67820

Disponível em: https://www.nucleodoconhecimento.com.br/salute/cancro-cervicale 
Figura 4 Mostra la percentuale di pap smear test (PCCU) eseguiti in Brasile tra il 2010 e il 2014, mediante scolarizzazione.

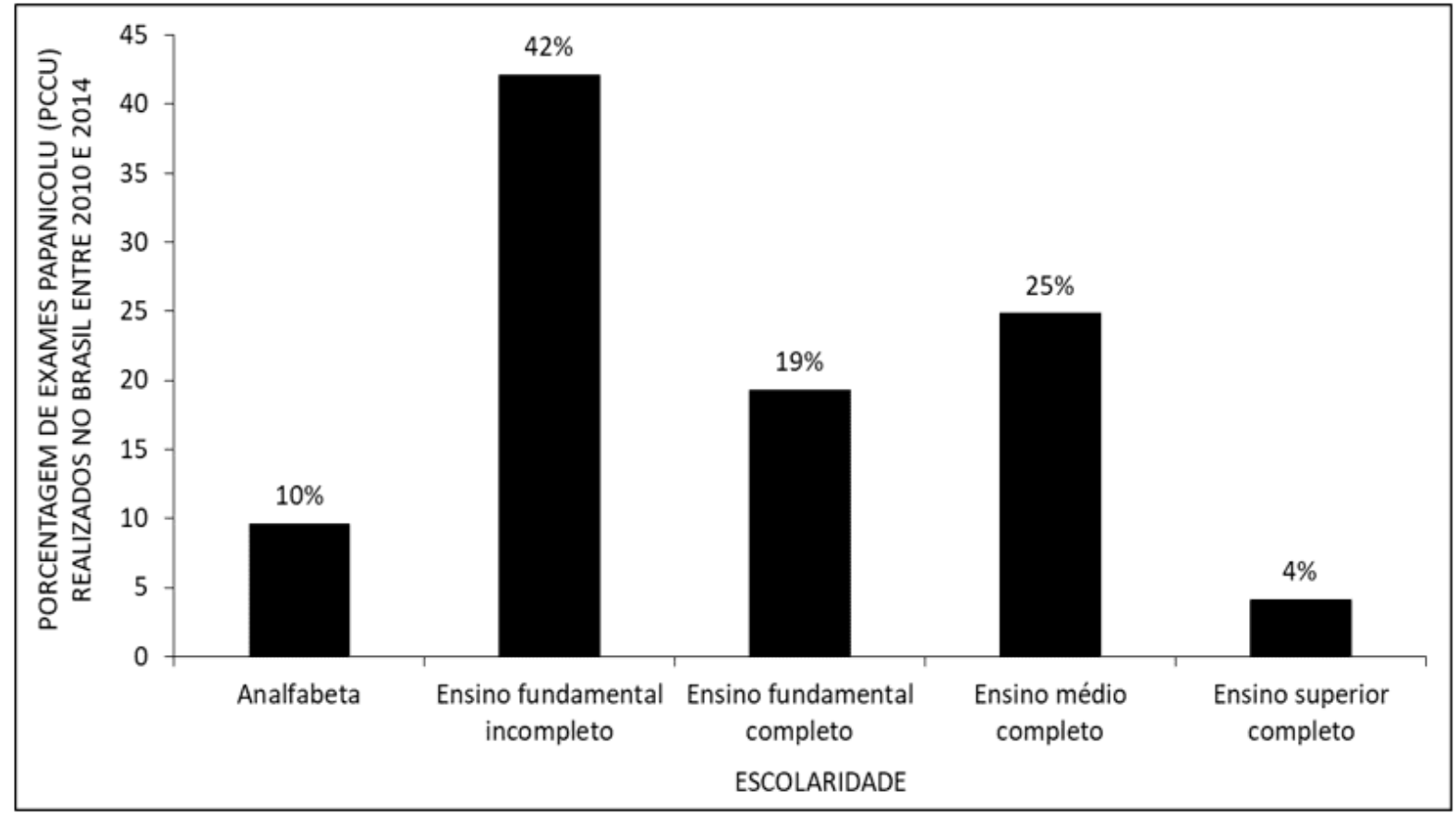

La figura 5 mostra la percentuale di scansioni pap smear (PCCU) eseguite in Brasile tra il 2010 e il 2014, per etnia. Le donne di etnia bianca e marrone eseguono un gran numero di test rispetto alle donne nere, gialle e indigene.

RC: 67820

Disponível em: https://www.nucleodoconhecimento.com.br/salute/cancro-cervicale 
La figura 5 Mostra la percentuale di scansioni pap smear (PCCU) eseguite in Brasile tra il 2010 e il 2014, per etnia.

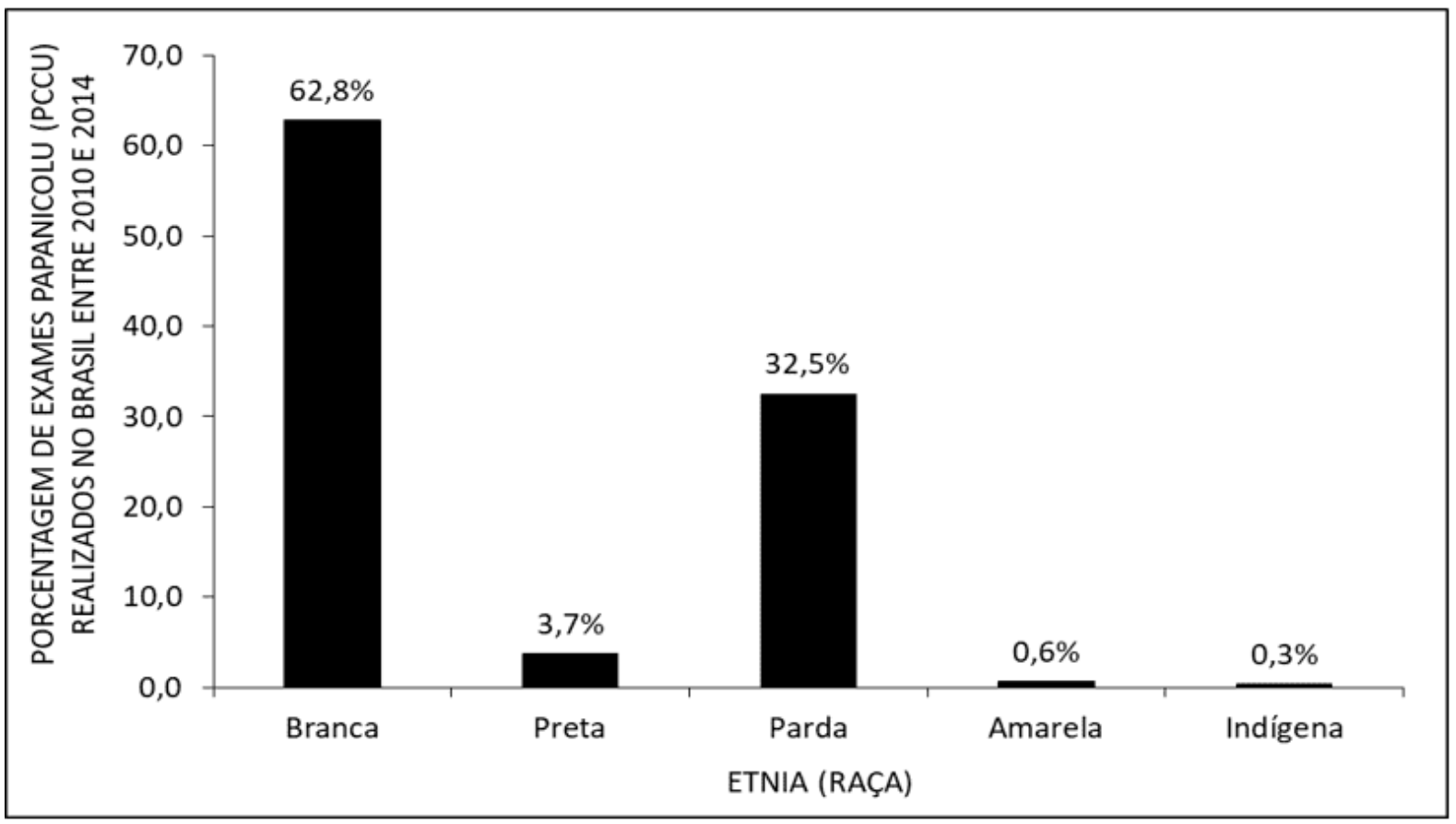

La figura 6 mostra la percentuale di scansioni pap smear eseguite in Brasile, nell'intervallo normale, tra il 2010 e il 2014. Tra i test eseguiti, i dati mostrano che la maggior parte non rientra nell'intervallo normale.

RC: 67820

Disponível em: https://www.nucleodoconhecimento.com.br/salute/cancro-cervicale 
La figura 6 Mostra la percentuale di pap smear test eseguiti in Brasile, nell'intervallo normale, tra il 2010 e il 2014.

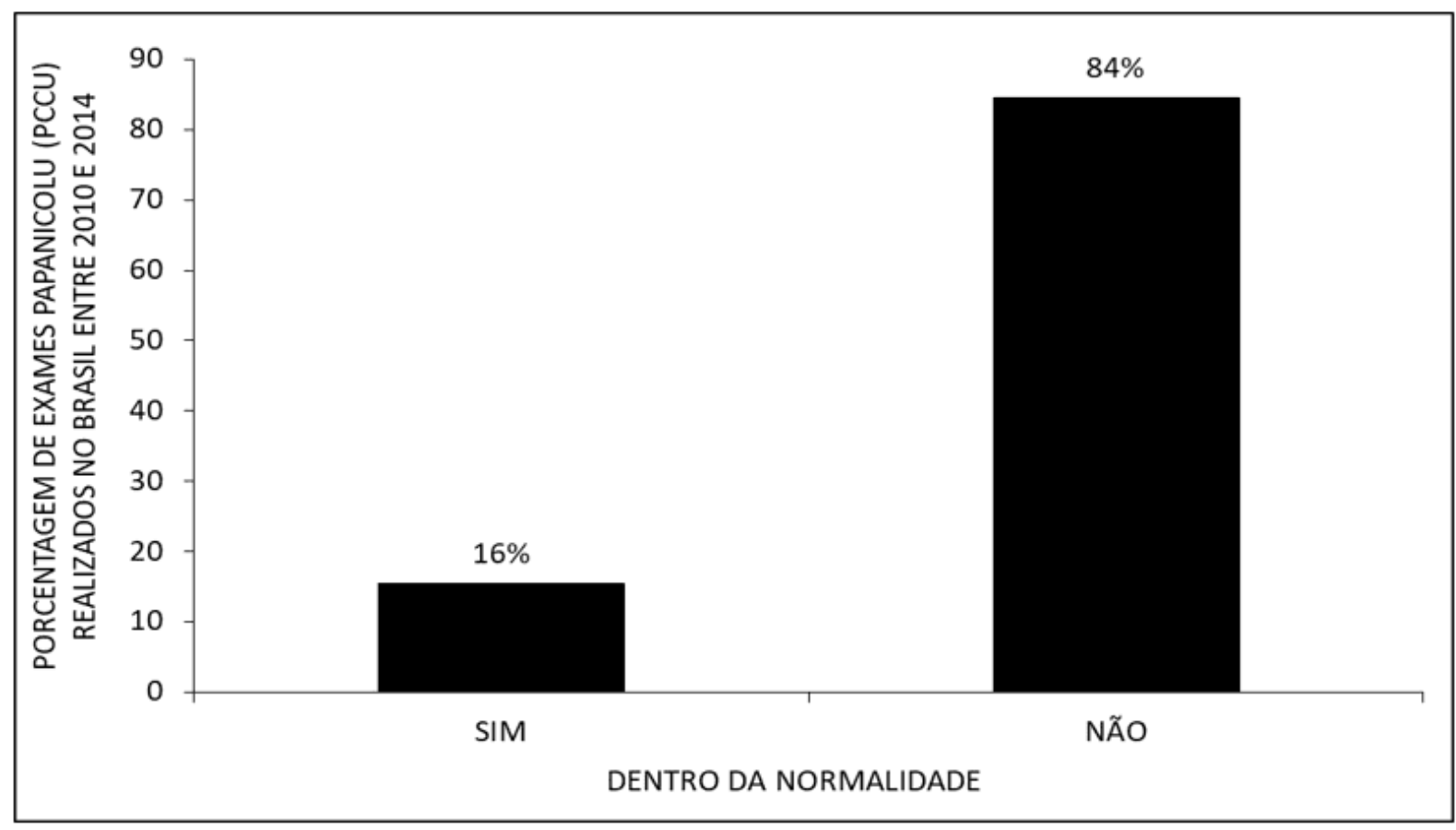

La figura 7 mostra la percentuale di scansioni pap smear (PCCU) eseguite in Brasile tra il 2010 e il 2014, per intervallo di raccolta. I test sono stati raccolti principalmente nell'intervallo fino a 10 giorni, mentre la quantità più piccola ha un intervallo di raccolta superiore a 30 giorni.

RC: 67820

Disponível em: https://www.nucleodoconhecimento.com.br/salute/cancro-cervicale 
Figura 7 Mostra la percentuale di pap smear test (PCCU) eseguiti in Brasile tra il 2010 e il 2014, per intervallo di raccolta.

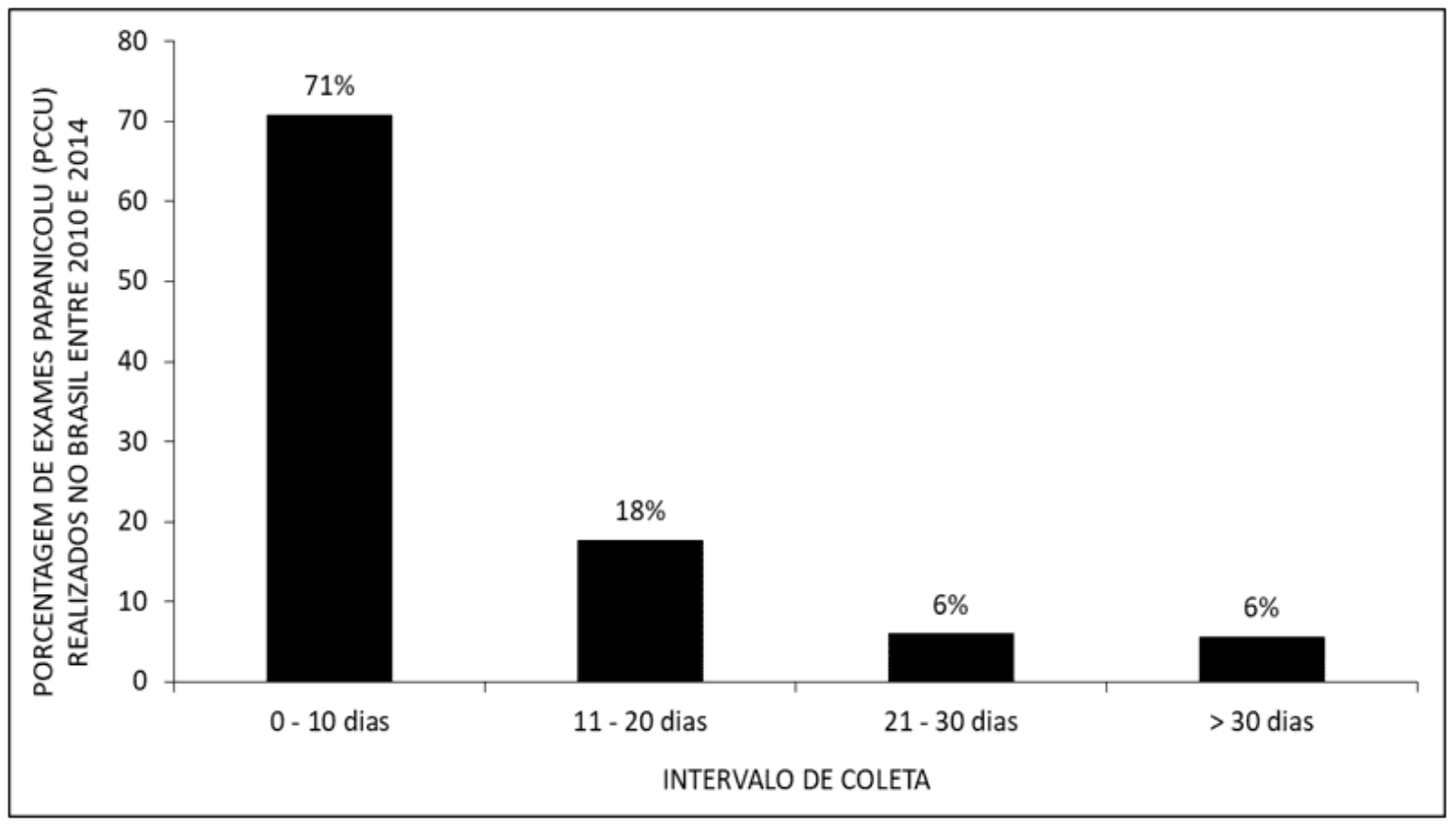

La figura 8 mostra la percentuale di scansioni pap smear (PCCU) eseguite in Brasile tra il 2010 e il 2014, per intervallo di risultati. Mostrando che la maggior parte dei risultati sono stati rilasciati entro 10 giorni. Mentre la quantità più piccola in più di 30 giorni.

RC: 67820

Disponível em: https://www.nucleodoconhecimento.com.br/salute/cancro-cervicale 
La figura 8 Mostra la percentuale di pap smear test (PCCU) eseguiti in Brasile tra il 2010 e il 2014, per intervallo di risultati.

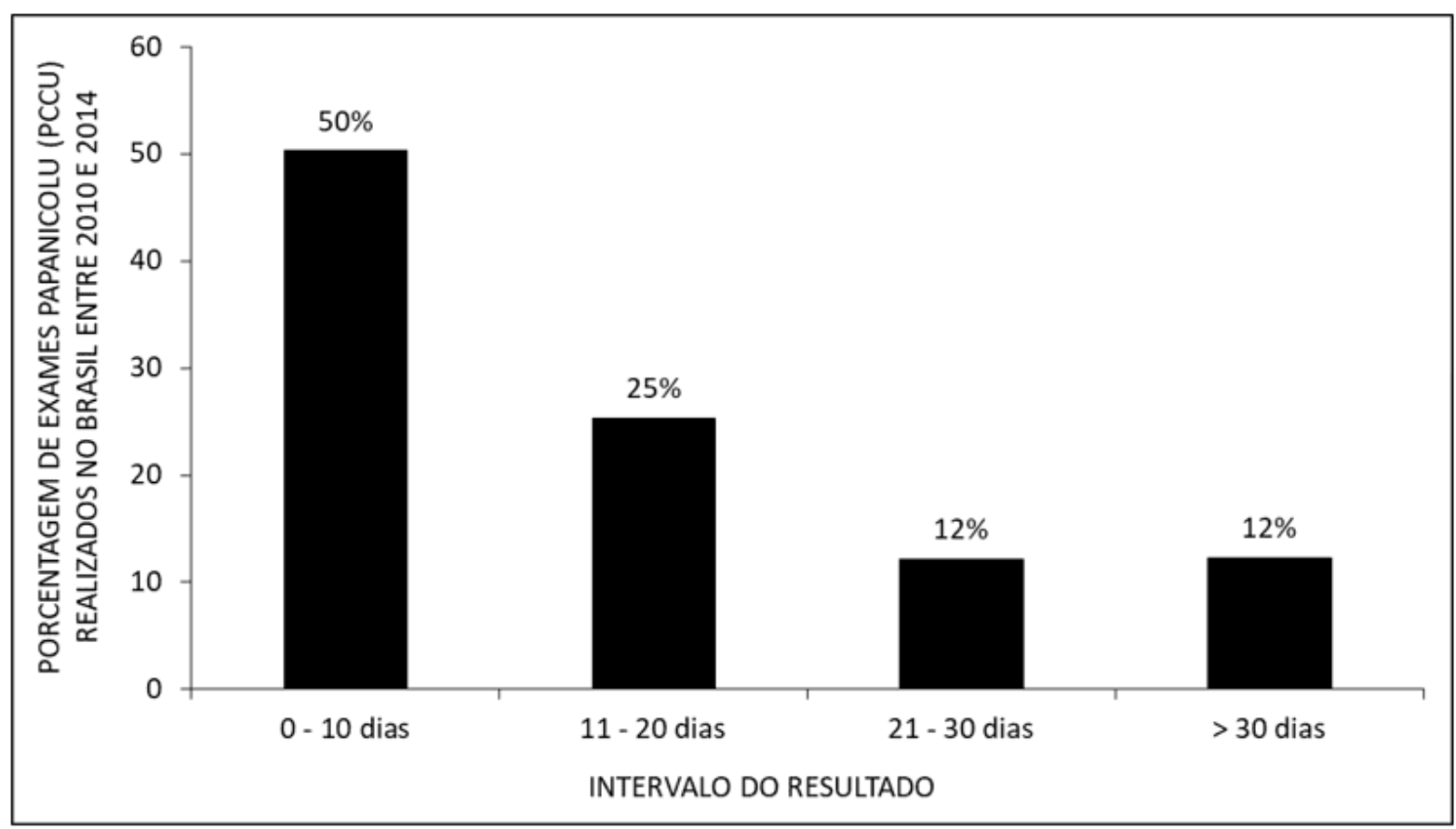

La figura 9 mostra la percentuale di scansioni pap smear (PCCU) eseguite in Brasile tra il 2010 e il 2014, secondo un precedente esame citologico. La maggior parte delle donne intervistate era stata precedentemente sottoposta a test.

RC: 67820

Disponível em: https://www.nucleodoconhecimento.com.br/salute/cancro-cervicale 
La figura 9 Mostra la percentuale di scansioni pap smear (PCCU) eseguite in Brasile tra il 2010 e il 2014, secondo il precedente esame citologico.

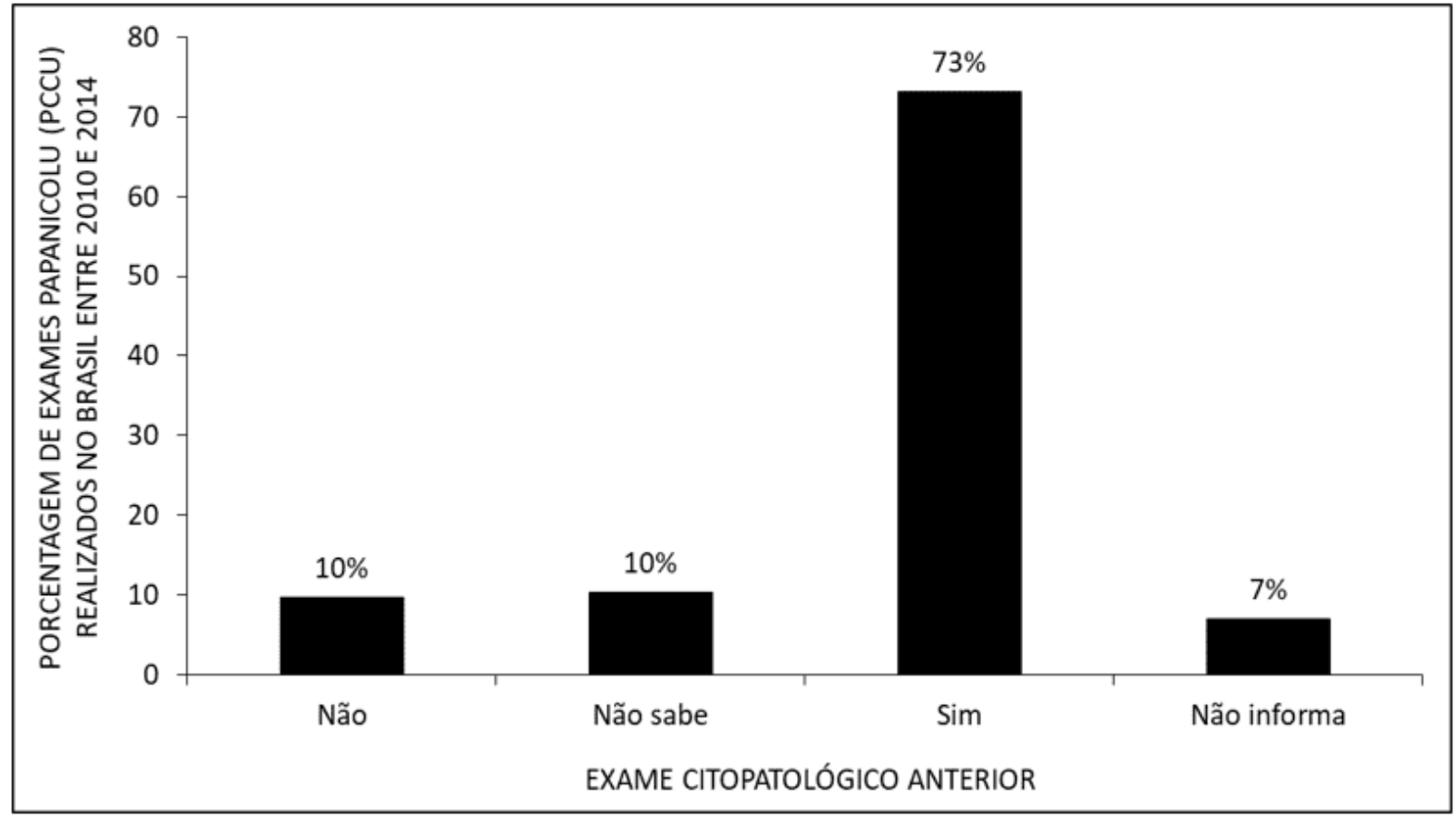

La figura 10 mostra la percentuale di scansioni pap smear (PCCU) eseguite in Brasile tra il 2010 e il 2014, secondo il momento dell'ultimo preventivo. I dati mostrano che la maggior parte delle donne partecipa agli esami di anno in anno.

RC: 67820

Disponível em: https://www.nucleodoconhecimento.com.br/salute/cancro-cervicale 
La figura 10 mostra la percentuale di pap smear test (PCCU) eseguiti in Brasile tra il 2010 e il 2014, secondo l'ora dell'ultimo preventivo.

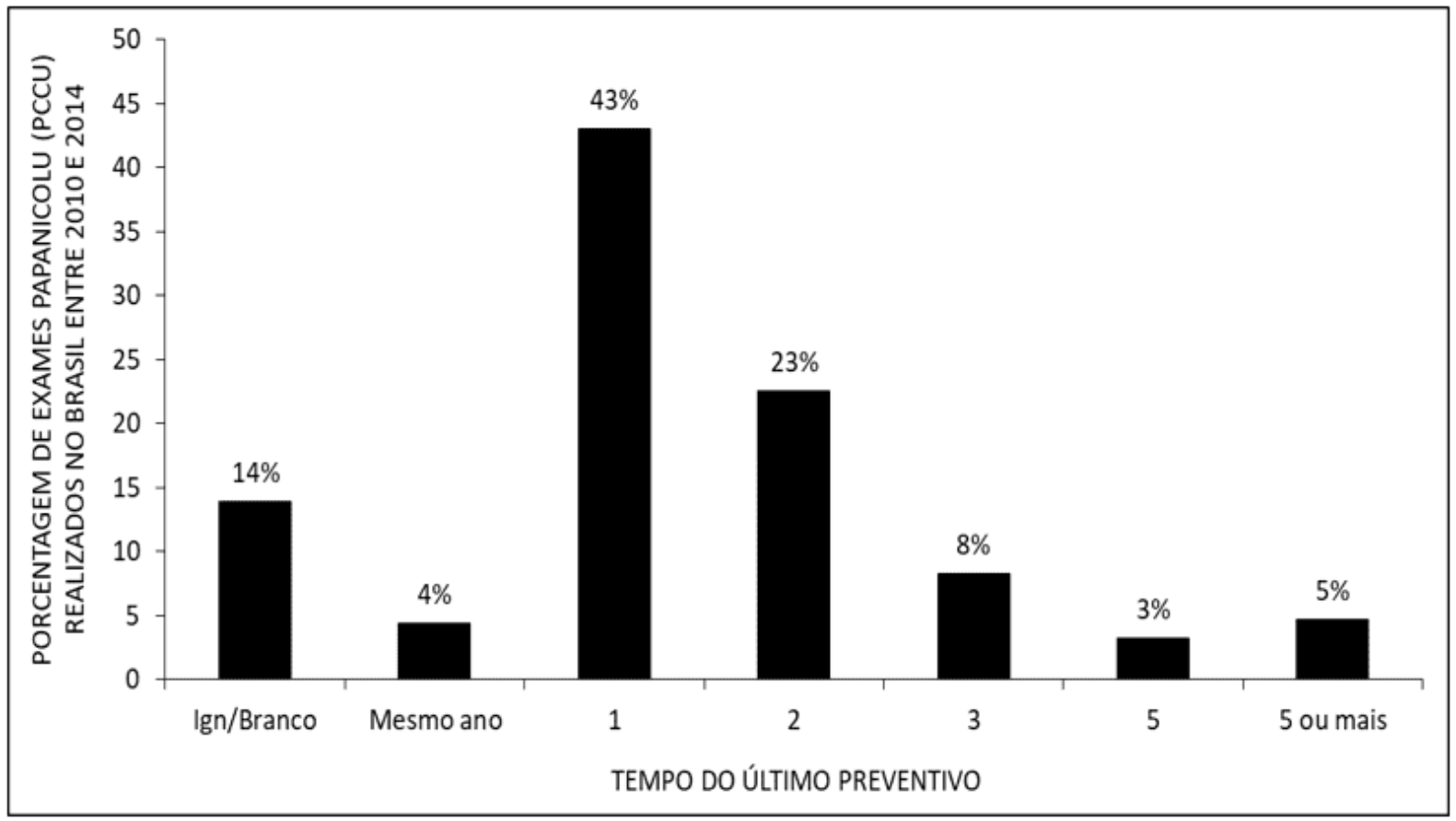

\section{DISCUSSIONE}

I dati mostrano una marcata diminuzione delle prestazioni dei test nel paese (figura 1). Alcuni fattori sembrano influenzare questa diminuzione, come la mancanza di conoscenza dell'importanza dell'esame e il timore dell'esame; vergogna dell'esposizione degli organi genitali; difficoltà di accesso agli UBS; mancanza di preparazione dei professionisti per quanto riguarda il rispetto, la guida e le informazioni fornite ai pazienti sull'esame (SANTOS e VARELA, 2015).

I dati mostrano un tasso più elevato di esami nella regione sud-orientale e un numero inferiore di test rispettivamente nelle regioni nord-orientale, meridionale, midwest e nord (figura 2). Ciò si basa sulla conoscenza, l'informazione e la consapevolezza delle donne nel trattamento della prevenzione del cancro cervicale. Nelle altre regioni, vi è stata una bassa positività dei test. Di conseguenza, Amapá, che si trova nella regione settentrionale, ha trovato meno test che hanno raggiunto una

$\mathrm{RC}: 67820$

Disponível em: https://www.nucleodoconhecimento.com.br/salute/cancro-cervicale 
percentuale inferiore all' $1,0 \%$ di positività. Così come nelle regioni nord-orientale, meridionali e del Midwest. Si è notato che queste regioni non hanno avuto partecipazione e consapevolezza delle donne, perché la scarsa conoscenza scientifica dello svolgimento dell'esame è un fattore che, in ogni caso, può ostacolare la ricerca dell'esame pccu. Alcune ragioni giustificano questo fatto, come la vergogna, il sentimento di paura, l'imbarazzo durante l'esame, la mancanza di tempo, la mancanza di interesse e la mancanza di informazioni sull'esame citopatologico (BORTOLON et al., 2012).

I dati mostrano che il maggior numero di test viene eseguito tra le donne di età compresa tra 30 e 39 anni e il numero più basso tra le donne fino a 19 anni (figura 3). L'inizio della raccolta degli esami dovrebbe essere a 25 anni indipendentemente dal fatto che il paziente abbia o meno una vita sessuale attiva. Una grande quantità di pap smear viene eseguita nelle fasce d'età da 30 a 39 anni, secondo la tabella. L'esame deve seguire fino all'età di 64 anni e deve essere interrotto dopo aver eseguito due prove i cui risultati sono negativi, due prove devono essere eseguite con tempi di intervallo da uno a tre anni. Tutte le donne che hanno una vita sessuale attiva o di età superiore ai 18 anni sono in grado di eseguire l'esame preventivo pccu, ma questo test non è ancora completamente acquisito dalle donne brasiliane, è noto che il Brasile ha un alto tasso di mortalità per cancro cervicale. Questo perché la maggior parte delle donne cerca un trattamento in misura più avanzata, che si trova più spesso nelle donne di età compresa tra 30 e 39 anni. Secondo la tabella 3 , è stato analizzato che il numero più basso di test eseguiti è tra le donne di 19 anni, proprio a causa della mancanza di informazioni sull'esame pccu e della paura di eseguito (BRASIL, 2014).

La maggior parte delle donne che hanno seguito l'esame ha una scuola elementare incompleta e un'istruzione superiore minoritaria (figura 4). In questa tabella è stato osservato che più studi e istruzione scolastica hanno le donne, meno sono interessate a svolgere l'esame preventivo pccu. La ragione di questo risultato è legata alle credenze e agli atteggiamenti nella salute. II $29,6 \%$ ha dichiarato di non

RC: 67820

Disponível em: https://www.nucleodoconhecimento.com.br/salute/cancro-cervicale 
ricordare perché non ha partecipato alla prova. Alcune ragioni sono dovute alla paura, alla vergogna, al disagio e al dolore. Abbiamo ottenuto risultati inaspettati nella tabella 4, perché abbiamo notato un alto tasso di esami pccu eseguiti da donne che hanno una scuola elementare incompleta perché in generale, le donne che non hanno una scolarizzazione incompleta tendono a costruire famiglie prima e, di conseguenza, hanno più figli delle donne che hanno un livello di istruzione più elevato. Di conseguenza, queste donne vanno dal medico più frequentemente, ottenendo un alto tasso di pap smear (SILVA et al., 2015).

Le donne di etnia bianca e marrone eseguono un gran numero di test rispetto alle donne nere, gialle e indigene (figura 5). È stato notato che gran parte dello striscio non pap è associato all'etnia, e gran parte di questa resistenza è correlata a fattori come le disuguaglianze di razza / etnia, così come in relazione all'accesso limitato che una data razza ha. Come indicato nella tabella (5). Tuttavia, la scarsa copertura e la mancanza di accessibilità tra gli altri gruppi si spiega con l'accesso e il trattamento differenziato che hanno quando arrivano al luogo dell'esame (PINHO E JUNIOR, 2003).

I dati mostrano che la maggior parte dei test eseguiti non rientra nell'intervallo normale (figura 6). Gran parte della popolazione femminile è fuori dall'ordinario quando si esegue il pap smear. Questo perché le donne cercano di fare l'esame in uno stato leggermente più avanzato. Ciò è anche spiegato dal fatto che la maggior parte delle donne ha più di un partner sessuale, con la tendenza a contrarre l'HPV (papillomavirus umano), una malattia a trasmissione sessuale. Dove può esserci disorganizzazione cellulare nel tessuto che costituisce la cervice (NORONHA et al., 2005; OLIVEIRA et al., 2016)).

Le prove sono state per lo più raccolte nell'intervallo fino a 10 giorni e la quantità più piccola ha avuto un intervallo di raccolta superiore a 30 giorni (figura 7 ). L'intervallo di raccolta è più spesso più breve e ha una durata dell'intervallo di 10 giorni lavorativi. II professionista legge la lama e impressiona il risultato durante questo periodo, l'esame pccu viene raccolto durante una consultazione ginecologica e durante la

RC: 67820

Disponível em: https://www.nucleodoconhecimento.com.br/salute/cancro-cervicale 
consultazione viene inserito uno speculum vaginale senza alcuna azione lubrificante, che può causare un piccolo disagio. Prima di raccogliere il paziente non dovrebbe fare sesso il giorno prima. Dovrebbe essere eseguito al di fuori del periodo mestruale per ottenere un risultato più efficace (BRITO e NERY, 2007).

La maggior parte dei risultati è stata rilasciata entro 10 giorni e l'importo più basso in più di 30 giorni (figura 8). In relazione al pap smear, il 46,55\% delle donne è stato sottoposto al test. Tuttavia, il $57,8 \%$ si esibisce in reti private e i risultati dell'esame sono molto più rapidi, poiché i risultati delle donne che eseguono l'esame pccu da parte della rete SUS che ha un tempo di consegna più lungo del previsto (MURATA et al., 2012).

La maggior parte delle donne intervistate era stata precedentemente sottoposta a test (figura 9). Tra le donne che sono a conoscenza dell'esame PCCU, la maggior parte di loro esegue frequentemente l'esame o si è esibita prima. Credere che con l'esecuzione dell'esame pccu in determinati periodi eviterà, quindi, malattie future, come il cancro cervicale. Perché è un test efficace che se diagnosticato all'inizio, può avere una probabilità al 100\% di cura (BARBEIRO et al., 2009).

I dati mostrano che la maggior parte delle donne partecipa agli esami di anno in anno (figura 10). La maggior parte delle donne va dal medico per eseguire l'esame del cancro cervicale con l'intenzione di prevenire le malattie e curarle all'inizio, per evitare problemi in futuro. Si raccomanda di eseguire l'esame pccu annualmente, di anno in anno, per analizzare e valutare se c'è stata o si è verificata qualche alterazione come è stato il risultato dell'esame precedente. Le donne che hanno a che fare con la loro salute vogliono l'esame con periodi di tempo da 1 a due anni, come mostrato nella figura (10) (PINHO e JUNIOR, 2003; MELO et al., 2019).

\section{CONCLUSIONE}

Questo studio ha dimostrato che quando c'è una buona prestazione per prevenire una malattia considerata grave, il risultato è soddisfacente ed efficace. È importante

RC: 67820

Disponível em: https://www.nucleodoconhecimento.com.br/salute/cancro-cervicale 
che abbiano informazioni e informazioni sull'esame preventivo, ma è noto che la prevenzione stessa viene eseguita solo con le prestazioni del pap smear per rilevare precocemente il livello del grado di cancro. Che se trattato all'inizio ha un'alta possibilità di cura.

Attraverso i risultati ottenuti analizzando i dati tra gli anni 2010 e 2014, rispettivamente, si conclude che nel corso degli anni la ricerca del test è gradualmente aumentata, ma alcuni aspetti sono persistito, come la vergogna di eseguire il test pcc, la paura, il dolore, tra gli altri. Tuttavia, i risultati hanno mostrato un alto tasso di esami nella regione sud-orientale e un indice più basso nella regione settentrionale. I dati mostrano una scarsa divulgazione e una scarsa consapevolezza per le donne nella regione settentrionale. Pertanto, la diffusione, la consapevolezza e le prestazioni dell'esame citopatologico sono molto importanti per la prevenzione del cancro cervicale, che oggi è la principale causa di mortalità tra le donne brasiliane

\section{RIFERIMENTI}

ARAÚJO, R.M.; CUNHA, H.C.O.; FERREIRA, V.G.C.; TRINDADE, M.P.; SOUZA, H.S.L. a importância da realização do exame papanicolau como instrumento de prevenção do câncer de colo do útero: uma revisão integrativa de literatura, Anais do V Congresso de Educação em Saúde da Amazônia. COESA. Universidade Federal do Pará. 2359-084. 2016.

BARBEIRO, F.M.S.; CORTEZ, E.A.; OLIVEIRA, P.A.M.C.; SILVA, A.L.O.; conhecimentos e práticas das mulheres acerca do exame papanicolau e prevenção do câncer cérvico-uterino. Cuidado é fundamental. 1(2):414-422. 2009.

BORTOLON, P.C.; SILVA, M.A.F.; CORRÊA, F.M.; DIAS, M.B.K.; KNUPP, V.M.A.; ASSIS, A.; CLARO, I.B. Avaliação da Qualidade dos Laboratórios de Citopatologia do Colo do Útero no Brasil. Revista Brasileira de Cancerologia. 58(3),435444,2012 .

RC: 67820

Disponível em: https://www.nucleodoconhecimento.com.br/salute/cancro-cervicale 
BRASIL. Ministério da Saúde. Secretaria de Atenção à Saúde. Departamento de Atenção Básica. Controle dos cânceres do colo do útero e da mama / Ministério da Saúde, Secretaria de Atenção à Saúde, Departamento de Atenção Básica. - 2. ed. - Brasília: Editora do Ministério da Saúde, 2014. 124 p.

BRASIL. Ministério da Saúde. Secretaria de Atenção à Saúde. Departamento de Atenção Básica. Controle dos cânceres do colo do útero e da mama. Ministério da Saúde. 2. ed. - Brasília: Editora do Ministério da Saúde. 124 p. 2013.

MELO, Carolina Simas. Et al. Caracterização epidemiológica dos óbitos no Brasil por macrorregião de 2016 a 2018. Revista Científica Multidisciplinar Núcleo do Conhecimento. Ano 04, Ed. 12, Vol. 01, pp. 05-17, 2019. Link de acesso: https://www.nucleodoconhecimento.com.br/saude/obitos-no-brasil

MENETRIER, J.V.; BOING, A.; MEDEIROS, K.A. Alterações citopatológicas do colo uterino em mulheres atendidas na 8a Regional de Saúde do Paraná no ano de 2014. Espaço para a Saúde - Revista de Saúde Pública do Paraná. V. 17, n. 2, p. 169177, 2016.

MURATA, I.M.H.; GRABRIELLONI, M.C.; SCHIRMER J. Cobertura do Papanicolau em mulheres de 25 a 59 anos de Maringá - PR, Brasil. Rev Bras

Cancerol.58(3):409-15, 2012.

NORONHA, V.L.; NORONHA, R.; CARMONA, B.; MACEDO, L.A.; CRUZ, E.M.; NAUM, C.; MELLO, W.; VILLA, L. Papilomavírus humano (hpv) em mulheres com citologia oncótica dentro dos limites da normalidade. ARTICLE. 17(1): 49-55, 2005.

OLIVEIRA, R.C.E.. et. al. Perfil epidemiológico do HPV da população feminina sexualmente ativa, na faixa etária de 10 a 80 anos. Revista Científica Multidisciplinar Núcleo do Conhecimento. Ano 01, Ed. 07, Vol. 04, pp. 21-34, 2016. Link de acesso: https://www.nucleodoconhecimento.com.br/saude/hpv

RC: 67820

Disponível em: https://www.nucleodoconhecimento.com.br/salute/cancro-cervicale 
PINHO, A.A; JUNIOR, I.F. Prevenção do câncer de colo do útero: um modelo teórico para analisar o acesso e a utilização do teste de Papanicolaou. Rev. Bras. Saude Mater. Infant. n.1 Recife jan./mar. 2003.

RICO, A.M.; SILVA, G.A.; LOWY, I.; TEIXEIRA, L. (Org.) et al. Câncer de mama, câncer de colo de útero: conhecimentos, políticas e práticas. Outras Letras. 256 p. 2015.

SANTOS, A.C.S.; VARELA, C.D.S. Prevenção do câncer de colo uterino: motivos que influenciam a não realização do exame de papanicolaou. Revista Enfermagem Contemporânea. 4(2):179-188, 2015.

SILVA, M.A; TEIXEIRA, E.M.B; FERRARI, R.A.P; CESTARI, M.E.W; CARDELLI, A.M. Fatores relacionados a não adesão à realização do exame de Papanicolau. Rev Rene. 16(4).532-9. 2015.

Inviato: Novembre 2020.

Approvato: Novembre 2020.

RC: 67820

Disponível em: https://www.nucleodoconhecimento.com.br/salute/cancro-cervicale 